\title{
Effect of the Magnetic Field Model on Cosmic Ray Coupling Coefficient Calculations
}

\author{
C. P. BAKER ${ }^{1}$, J. E. Humble ${ }^{1}$, and M. L. DULDig ${ }^{2}$ \\ ${ }^{1}$ Physics Department, University of Tasmania, Hobart, Tasmania 7001, Australia \\ ${ }^{2}$ Australian Antarctic Division, Kingston, Tasmania 7050, Australia
}

(Received October 2, 1989; Revised January 31, 1990)

\begin{abstract}
The relationship between primary cosmic ray particles in space and the associated secondary particles observed using ground-based detectors can be described by a set of coupling coefficients. These coefficients depend, among other things, on the direction that the detector is pointing through the geomagnetic field. Along certain directions the coefficients may be highly sensitive to small changes in the field. The accuracy of the field model thus places restrictions on the accuracy of the coupling coefficients. Small errors in the field model can give large relative errors in the coupling coefficients. This will consequently give large relative errors in the interpretation of the space distribution of the primary particles inferred from measured data.
\end{abstract}

\section{Introduction}

The ultra-high energy charged particles which bombard the earth's atmosphere from space are known as primary cosmic ray particles. Around $90 \%$ of all primary particles are protons and $9 \%$ are Helium nuclei. The intensity distribution of these particles in space is almost isotropic. Small and systematic directional variations are however observed. These variations are normally of the order of a few tenths of a percent but nevertheless are physically significant. They are caused by the interplanetary electromagnetic field, the solar wind and the motion of the earth through space. By studying any anisotropy, or three-dimensional directional variation in intensity, information on the electromagnetic field structure of interplanetary space can be inferred.

Most of the primary particles never reach sea-level. They interact with nuclei in the earth's atmosphere to produce secondary cosmic radiation. A significant secondary component consists of muons which are produced from the decay of pions. The pions are a direct product of reactions between primary particles and atmospheric nuclei.

By measuring the intensity variation of the muons, using ground-based muon detectors, the three-dimensional space distribution of the primary particles can be calculated. The type of muon detector considered here consists of two rectangular trays with a fixed separation. Within each tray is a number of particle detectors such that if a muon passes through both trays, i.e. is within the field of view subtended by the overlap of the trays, it is counted. The detector is fixed with respect to the terrestrial frame of reference and hence sees a small daily variation in intensity of muons as the earth rotates about its axis.

The relationship between the distributions of primary particles and muons can be described by a set of so-called coupling coefficients. To calculate these coefficients 
asymptotic directions are required for every possible incident direction of secondary particle passing through the detector. The asymptotic direction of a primary particle, as illustrated in Fig. 1, is that direction from which it was travelling before being influenced by the earth's magnetic field. Due to the high energies of the primary particles, a muon effectively travels in the same direction as that of the primary particle from which it was produced. To calculate the asymptotic directions, data describing the earth's magnetic field are employed from the height of the detector to free space.

Small changes in the geomagnetic field model will give small changes in the calculated asymptotic directions. For particular telescope configurations these small changes may give large relative changes in the coupling coefficients. In such circumstances the accuracy of the field model limits the accuracy of the coupling coefficients. The observed intensity variation and the space distribution are related linearly through the coupling coefficients and therefore a factor of two change in the coefficients, for example, will give rise to a factor of two change in the inferred space distribution.

The formalism used in this paper to describe the three-dimensional intensity distribution of primary particles in space, and the subsequent definition of the coupling coefficients, was originally developed by NAGASHIMA (1971). Coupling coefficients have been calculated by FUJIMOTO et al. (1984) and we use an extended version of their method (BAKER et al., 1989). Nagashima's formalism is outlined below in order to illustrate the use of the magnetic field data and the sensitivity of coupling coefficients in some circumstances.

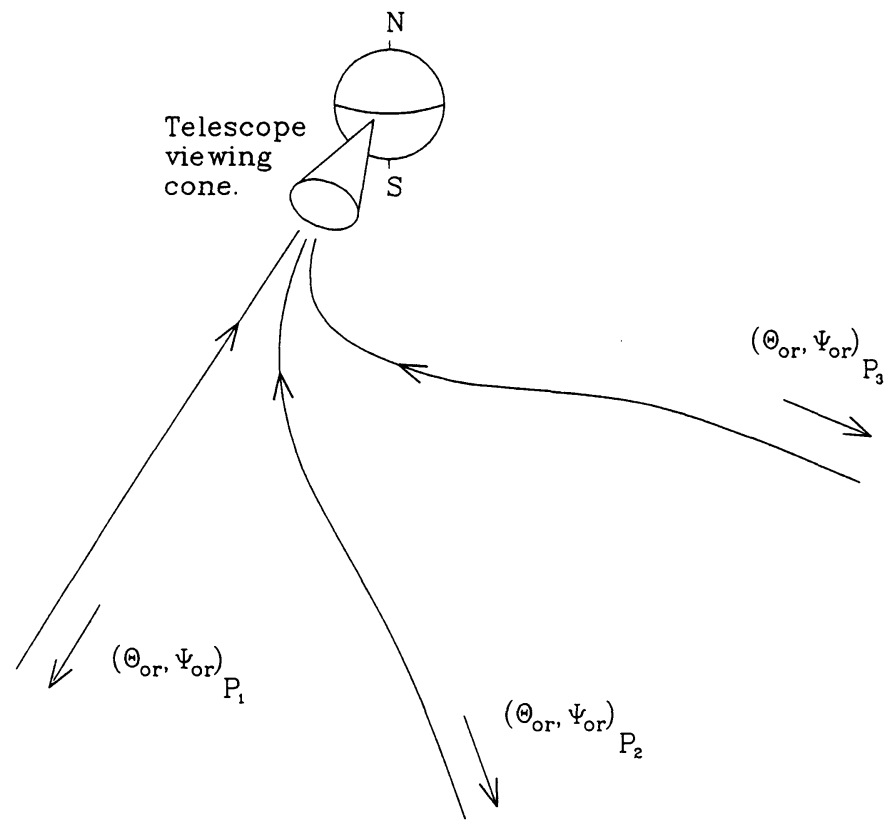

Fig. 1. Asymptotic directions $\left(\theta_{\mathrm{or}}, \psi_{\mathrm{or}}\right)$ are shown for three primary particle trajectories entering the field of view of the telescope. The trajectories are a function of particle rigidity $P$. In this case, $P_{1} \gg P_{2}>P_{3}$. 


\section{Formalism}

Let the flux per steradian per second of primary particles with rigidity $P$, moving in a direction in space defined by polar angle $\chi$ and azimuth angle $\Lambda$ relative to an arbitrary coordinate system, be described by the function $J(P, \chi, \Lambda)$. Rigidity is relativistic momentum per unit charge and has units of volts.

Since any variation of $J$ with $(\chi, \Lambda)$ will be small we can write

$$
J(P, \chi, \Lambda)=J(P)+\delta J(P, \chi, \Lambda)
$$

where $J(P)$ is the isotropic flux. Since the anisotropy is three-dimensional, it is convenient to express the degree of anisotropy $\delta J / J$ as a series of axi-symmetric anisotropies. For a single axis-symmetric anisotropy, with the axis of symmetry corresponding to $\chi=0$,

$$
\frac{\delta J(P, \chi, \Lambda)}{J(P)}=F(\chi) \cdot G(P)
$$

$G(P)$ is the differential rigidity spectrum of the anisotropý and is adequately described by the power-law:

$$
G(P)= \begin{cases}(P / 10)^{\gamma} & P \leq P_{\mathrm{u}}, \\ 0 & P>P_{\mathrm{u}},\end{cases}
$$

for constants $\gamma, P_{\text {u }}$ (FUjImoto et al., 1984). $F(\chi)$ is called the space distribution. To ease co-ordinate conversion to a terrestrial frame of reference it is convenient to expand $F(\chi)$ in a series of Legendre polynomials. The transformation to geographic coordinates then yields

$$
F(\chi)=\sum_{n=0}^{\infty} \sum_{m=0}^{n} f_{n}^{m}(\chi)
$$

where

$$
f_{n}^{m}(\chi)=\eta_{\mathrm{n}} P_{n}^{m}\left(\cos \theta_{\mathrm{R}}\right) P_{n}^{m}\left(\cos \theta_{\mathrm{J}}\right) \cos m\left(\alpha_{\mathrm{J}}-\alpha_{\mathrm{R}}\right) .
$$

Here $\eta_{\mathrm{n}}$ are constants, $\left(\alpha_{\mathrm{R}}, \theta_{\mathrm{R}}\right)$ are the right-ascension and declination of the symmetry axis of the anisotropy, $\left(\alpha_{\mathrm{J}}, \theta_{\mathrm{J}}\right)$ are the right-ascension and declination of the direction corresponding to $(\chi, \Lambda)$ in space, and the functions $P_{n}^{m}(x)$ are the semi-normalized spherical functions (CHAPMAN and BARTELS, 1940) related to the ordinary associated Legendre functions $P_{n, m}(x)$ by

$$
P_{n}^{m}(x)= \begin{cases}P_{n, m}(x) & m=0 \\ \left\{\frac{2(n-m) !}{(n+m) !}\right\}^{1 / 2} P_{n, m}(x) & m \neq 0\end{cases}
$$


The space distribution $F(\chi)$ produces a daily variation $D(t)$ in the intensity of muons as seen from a muon detector fixed in its local frame of reference as the earth rotates daily. Each component $f_{n}^{m}(\chi)$ produces an observed variation $D_{n}^{m}(t)$ where

$$
D(t)=\sum_{n=0}^{\infty} \sum_{m=0}^{n} D_{n}^{m}(t)
$$

Let $D(t)$ be expanded in a Fourier series such that

$$
D_{n}^{m}(t)=A_{n}^{m} \cos (2 \pi m t)+B_{n}^{m} \sin (2 \pi m t) \quad(t \text { in days }) .
$$

$A_{n}^{m}$ and $B_{n}^{m}$ are the coefficients in the Fourier series expansion. Define $c_{n}^{m}$ and $s_{n}^{m}$ through

$$
\begin{aligned}
& A_{n}^{m}=c_{n}^{m} x_{n}^{m}+s_{n}^{m} y_{n}^{m}, \\
& B_{n}^{m}=-s_{n}^{m} x_{n}^{m}+c_{n}^{m} y_{n}^{m},
\end{aligned}
$$

where

$$
\begin{aligned}
& x_{n}^{m}=\eta_{\mathrm{n}} P_{n}^{m}\left(\cos \theta_{\mathrm{R}}\right) \cos \left(2 \pi m t_{\mathrm{R}}\right), \\
& y_{n}^{m}=\eta_{\mathrm{n}} P_{n}^{m}\left(\cos \theta_{\mathrm{R}}\right) \sin \left(2 \pi m t_{\mathrm{R}}\right),
\end{aligned}
$$

$t_{\mathrm{R}}$ is the local time representation of $\alpha_{\mathrm{R}}$. Let $S_{n}^{m}(t)$, called the space harmonic variation, be defined by

$$
\begin{aligned}
S_{n}^{m}(t) & =x_{n}^{m} \cos (2 \pi m t)+y_{n}^{m} \sin (2 \pi m t) \\
& =\eta_{\mathrm{n}} P_{n}^{m}\left(\cos \theta_{\mathrm{R}}\right) \cos \left(2 \pi m\left[t-t_{\mathrm{R}}\right]\right) .
\end{aligned}
$$

Then from Eq. (2),

$$
f_{n}^{m}(\chi)=P_{n}^{m}\left(\cos \theta_{\mathrm{J}}\right) S_{n}^{m}(t)
$$

where

$$
\begin{aligned}
& d_{n}^{m}=\sqrt{\left(c_{n}^{m}\right)^{2}+\left(s_{n}^{m}\right)^{2}}, \\
& \delta t_{n}^{m}=\frac{1}{2 m \pi} \tan ^{-1}\left(\frac{s_{n}^{m}}{c_{n}^{m}}\right) .
\end{aligned}
$$

From these relationships

$$
D_{n}^{m}(t)=d_{n}^{m} S_{n}^{m}\left(t+\delta t_{n}^{m}\right)
$$

Hence the space distribution can be derived from $S_{n}^{m}(t)$ through Eq. (3) which can in turn be derived from the components of the observed daily variation $D_{n}^{m}(t)$ and a knowledge of $c_{n}^{m}$ and $s_{n}^{m} . c_{n}^{m}$ and $s_{n}^{m}$ are called the coupling coefficients and relate the observed variation $D(t)$ to the space distribution $F(\chi)$. It can be shown (NAGASHIMA, 1971) that 


$$
\begin{aligned}
& c_{n}^{m}=\frac{1}{I} \int_{P_{\mathrm{s}}}^{\infty} \int_{\Omega} Y \cdot A \cdot G(P) \cdot P_{n}^{m}\left(\cos \theta_{\mathrm{or}}\right) \cdot \cos m\left(\psi_{\mathrm{or}}-\psi_{\mathrm{st}}\right) \mathrm{d} \omega \mathrm{d} P, \\
& s_{n}^{m}=\frac{1}{I} \int_{P_{\mathrm{c}}}^{\infty} \int_{\Omega} Y \cdot A \cdot G(P) \cdot P_{n}^{m}\left(\cos \theta_{\mathrm{or}}\right) \cdot \sin m\left(\psi_{\mathrm{or}}-\psi_{\mathrm{st}}\right) \mathrm{d} \omega \mathrm{d} P,
\end{aligned}
$$

where $I$ is the total intensity of observed muons, $Y$ is the muon response function which gives the number of muons arriving along each direction $(\theta, \psi)$ produced by primary particles of rigidity $P .(\theta, \psi)$ are respectively the local zenith and azimuth angles defining the incident direction. $P_{\mathrm{c}}$ is the cut-off rigidity below which $Y$ is insignificant. A gives the relative overlap of the two detector trays of the muon telescope along each direction $(\theta, \psi) . \Omega$ is the solid angle subtended by the two trays and $\mathrm{d} \omega$ is the element of solid angle. The integrals are over all rigidity values for which primary particles produce detectable muons and over all incident directions $(\theta, \psi)$ for which muons can enter the trays. $\psi_{\text {st }}$ is the geographic longitude of the telescope site and $\left(\theta_{\mathrm{or}}, \psi_{\mathrm{or}}\right)$ are respectively the geographic co-latitude and longitude defining the asymptotic direction of primary particles which produce muons with incident direction $(\theta, \psi)$. These directions are functions of the telescope site and the rigidity of the primary particles. A modified version of a trajectory tracing program by SHEA et al. (1965) was used to calculate the asymptotic directions needed to compute $c_{n}^{m}$ and $s_{n}^{m}$. The method of calculation involves integrating the trajectory of a negatively charged particle from the telescope site backwards along $(\theta, \psi)$, through the earth's magnetic field, using a Runge-Kutta method. The 1980 international geomagnetic field model was used (IAGA DIVISION 1, WORKING GROUP 1, 1986).

Each particular telescope configuration thus has its own set of coupling coefficients defined by Eqs. (4) and (5) which relate the observed variation in the intensity of muons to the directional distribution of primary cosmic ray particles in space.

\section{Analysis}

In some circumstances a small systematic directional change in the geomagnetic field model may give large relative changes in the coupling coefficients of a muon telescope. This occurs for coefficients $c_{j}^{k}, s_{j}^{k}$ when the telescope is pointing in a direction such that a significant fraction of the primary particles, which produce muons that are subsequently detected, have asymptotic co-latitudes $\theta_{\text {or }}$ such that $P_{j}^{k}\left(\cos \theta_{\text {or }}\right) \approx 0$. Note that the effect of a systematic directional change in the field model on the calculation of asymptotic directions for a particular muon telescope is indistinguishable from a corresponding change in viewing direction of that telescope.

To illustrate the sensitivity of some coefficients in the above circumstance we have calculated coupling coefficients for a muon telescope under the following conditions: cubic geometry (the length and width of the trays and the perpendicular separation between the trays are identical), location given by geographic latitude $-8^{\circ}$ and geographic longitude $0^{\circ}$, and depth underground equivalent to $30 \mathrm{hg} \cdot \mathrm{cm}^{-2}$ of absorbing material. At this depth the muon response function $Y$ has a maximum for rigidity $P \approx 50$ GV (Giga-Volt). At this geographic location primary particles of rigidity $50 \mathrm{GV}$ producing muons incident vertically have an asymptotic co-latitude $\theta_{\text {or }} \approx 0^{\circ}$. The coupling coefficients $c_{n}^{m}$ and $s_{n}^{m}$ for a telescope viewing vertically should be sensitive if $P_{n}^{m}\left(\cos \theta_{\mathrm{or}}\right)=$ $P_{n}^{m}(1)=0$. Some such coefficients are $c_{1}^{0}, s_{1}^{0}, c_{2}^{1}, s_{2}^{1}$ but not, for example, $c_{0}^{0}, c_{2}^{0}, c_{1}^{1}, s_{1}^{1}$. Note 
Table 1. Muon telescope coupling coefficients $\times 10^{4}$.

\begin{tabular}{|c|c|c|c|c|c|c|c|c|c|c|c|}
\hline \multirow{2}{*}{$\gamma$} & \multirow{2}{*}{$P_{\mathrm{u}}(\mathrm{GV})$} & \multicolumn{2}{|c|}{$c_{0}^{0}$} & \multicolumn{2}{|c|}{$c_{1}^{1}$} & \multicolumn{2}{|c|}{$s_{1}^{\prime}$} & \multicolumn{2}{|c|}{$c_{2}^{1}$} & \multicolumn{2}{|c|}{$s_{2}^{1}$} \\
\hline & & A & B & A & B & A & B & A & B & A & B \\
\hline 0.5 & 100 & 8806 & 8802 & 7181 & 7188 & 3443 & 3432 & -243 & 0 & 184 & 297 \\
\hline 0.5 & 200 & 18174 & 18172 & 15593 & 15621 & 5037 & 5018 & -1385 & -798 & 7 & 193 \\
\hline 0.5 & 500 & 30169 & 30171 & 26449 & 26515 & 5999 & 5978 & -3250 & -2195 & -138 & 94 \\
\hline 0.0 & 100 & 3618 & 3616 & 2873 & 2874 & 1517 & 1511 & -61 & 34 & 120 & 167 \\
\hline 0.0 & 200 & 6111 & 6110 & 5111 & 5118 & 1947 & 1941 & -362 & -176 & 73 & 139 \\
\hline 0.0 & 500 & 8279 & 8278 & 7072 & 7086 & 2127 & 2119 & -697 & -427 & 46 & 121 \\
\hline-0.5 & 100 & 1545 & 1544 & 1183 & 1184 & 699 & 696 & -8 & 30 & 75 & 96 \\
\hline-0.5 & 200 & 2215 & 2215 & 1784 & 1786 & 817 & 813 & -88 & -26 & 63 & 88 \\
\hline-0.5 & 500 & 2614 & 2613 & 2145 & 2148 & 851 & 847 & -149 & -71 & 58 & 85 \\
\hline
\end{tabular}

Values in column A are for a cubic telescope at geographic latitude $-8^{\circ}$, longitude $0^{\circ}$, under $30 \mathrm{hg} \cdot \mathrm{cm}^{-2} \mathrm{of}$ absorbing material and viewing vertically. Values in column B are for the same telescope tilted $2^{\circ}$ to the north.

that the viewing direction of the telescope is defined as the direction of the perpendicular to the trays.

Table 1, column A, lists coefficients $c_{0}^{0}, c_{1}^{1}, s_{1}^{1}, c_{2}^{1}, s_{2}^{1}$ for a muon telescope in the above configuration viewing vertically in the local frame of reference. The coefficients are functions of $\gamma$ and $P_{\mathrm{u}}$. These variables define the rigidity dependence $G(P)$ of the anisotropy as in Eq. (1). Table 1, column B, lists the same coefficients for an identical telescope which has been tilted from the vertical through $2^{\circ}$ to the north. From the table it can be seen that coefficients $c_{0}^{0}, c_{1}^{1}, s_{1}^{1}$ have been relatively unaffected by this small change in viewing direction whereas $c_{2}^{1}, s_{2}^{1}$ have in most cases undergone large relative changes. This viewing direction is therefore sensitive for coefficients $c_{2}^{1}, s_{2}^{1}$ but insensitive for coefficients $c_{0}^{0}, c_{1}^{1}, s_{1}^{1}$.

The sensitivity of the coupling coefficients is most severe for telescopes that have (i) a narrow angle cone of viewing; (ii) primary particles arriving from latitudes which give a zero in $P_{n}^{m}\left(\cos \theta_{\text {or }}\right)$ while at the same time have rigidities near the maximum of the muon response function $Y$; and (iii) muons from such primary particles arriving perpendicular to the telescope trays. Table 2 shows the effect of widening the field of view of the telescope. Values in columns A and B are coupling coefficients for telescopes in the same configuration as those for Table 1 except that the perpendicular separation of the trays has been halved. The sensitivity of coefficients $c_{2}^{1}, s_{2}^{1}$ is still apparent but is not as severe.

It is clear that the coupling coefficient sensitivity problem is due to the choice of function used in the series expansion of $F(\chi)$. It occurs when the telescope is pointing in such a direction that the integrand in the formulae for the coupling coefficients is near zero. This causes the sensitive coefficients to subsequently have values close to zero. Any series expansion of $F(\chi)$ will give rise to sensitive coefficients for the same reason. The sensitive directions depend on the choice of function used in the expansion. The problem is of practical significance when searching for anisotropies in these directions. Any change in the coupling coefficients will give rise to a change of the same order in the derived space distribution. Hence when searching for anisotropies by viewing in these sensitive directions, the accuracy of the field model places limits on the smallest 
Table 2. Muon telescope coupling coefficients $\times 10^{4}$.

\begin{tabular}{|c|c|c|c|c|c|c|c|c|c|c|c|}
\hline \multirow{2}{*}{$\gamma$} & \multirow{2}{*}{$P_{\mathrm{u}}(\mathrm{GV})$} & \multicolumn{2}{|c|}{$c_{0}^{0}$} & \multicolumn{2}{|c|}{$c_{1}^{1}$} & \multicolumn{2}{|c|}{$s_{1}^{1}$} & \multicolumn{2}{|c|}{$c_{2}^{1}$} & \multicolumn{2}{|c|}{$s_{2}^{1}$} \\
\hline & & A & B & A & B & A & B & A & B & A & B \\
\hline 0.5 & 100 & 8234 & 8232 & 6407 & 6413 & 3149 & 3140 & -204 & -65 & 217 & 284 \\
\hline 0.5 & 200 & 17734 & 17732 & 14427 & 14449 & 4765 & 4739 & -1149 & -786 & 95 & 214 \\
\hline 0.5 & 500 & 30352 & 30352 & 25094 & 25149 & 5770 & 5739 & -2723 & -2033 & -19 & 137 \\
\hline 0.0 & 100 & 3351 & 3350 & 2546 & 2547 & 1371 & 1367 & -54 & 0 & 125 & 153 \\
\hline 0.0 & 200 & 5877 & 5876 & 4677 & 4683 & 1806 & 1800 & -303 & -190 & 93 & 134 \\
\hline 0.0 & 500 & 8155 & 8154 & 6603 & 6615 & 1994 & 1987 & -585 & -414 & 71 & 120 \\
\hline-0.5 & 100 & 1416 & 1415 & 1041 & 1041 & 622 & 621 & -9 & 12 & 73 & 84 \\
\hline-0.5 & 200 & 2094 & 2093 & 1612 & 1614 & 741 & 739 & -75 & -38 & 64 & 79 \\
\hline-0.5 & 500 & 2512 & 2512 & 1966 & 1969 & 777 & 775 & -127 & -79 & 60 & 77 \\
\hline
\end{tabular}

Values in column $\mathrm{A}$ are for a semi-cubic telescope at geographic latitude $-8^{\circ}$, longitude $0^{\circ}$, under 30 $\mathrm{hg} \cdot \mathrm{cm}^{-2}$ of absorbing material and viewing vertically. Values in column B are for the same telescope tilted $2^{\circ}$ to the north.

anisotropy which can be derived from observations.

Ideally the field model should be accurate enough to ensure that the error in terms $P_{n}^{m}\left(\cos \theta_{\text {or }}\right)$ is less than or equal to errors in the other terms which define the coupling coefficients. The accuracy of these terms is limited by the muon response function $Y$ which is not known to better than approximately $2 \%$ due to limited knowledge of the collision cross-sections of high energy primary particles with atmospheric nuclei. Hence the terms $P_{n}^{m}\left(\cos \theta_{\text {or }}\right)$ should be accurate to similar order. Changes in both the magnitude and direction of the geomagnetic field will affect $P_{n}^{m}\left(\cos \theta_{\text {or }}\right)$. As illustrated by the example given, a systematic error of $2^{\circ}$ in the field will give significant errors in some coupling coefficients if the telescope is pointing in a sensitive direction. In general, the required accuracy of the field model will depend critically on the particular muon telescope configuration and the coupling coefficient.

\section{Conclusion}

It has been shown through example that in some circumstances small changes in the viewing direction of a muon telescope can give large relative changes in the corresponding coupling coefficients and therefore large changes in the interpreted space distribution of primary particles. An order of magnitude error in the relevant coupling coefficients will cause a corresponding order of magnitude error in the derived space distribution due to the linear relationship between them. Since the asymptotic direction of a particle is a function of the geomagnetic field, such a change in viewing direction is indistinguishable from a small systematic directional change in the field model. The accuracy of these coefficients is therefore constrained to the accuracy of the field model.

\section{REFERENCES}

Baker, C. P., M. L. Duldig, and J. E. Humble, Proc. Astron. Soc. Aust., Vol. 8, No. 1, 55, 1989.

Chapman, S. and J. Bartels, Geomagnetism, Vol. II, 611 pp., Oxford Press, 1940. 
Fujimoto, K., A. Inoue, K. Murakami, and K. Nagashima, Coupling coefficients of cosmic ray daily variations for meson telescopes, Rep. 9, Cosmic Ray Res. Lab., Nagoya University, Nagoya, Japan, 1984.

IAGA Division 1, Working Group 1, International Geomagnetic Reference Field Revision 1985, Eos, Trans. Am. Geophys. Union, 67, 523, 1986.

Nagashima, K., Rep. Ionos. Space Res. Japan, 25, 189, 1971.

Shea, M. A., D. F. Smart, and K. G. McCracken, A study of vertical cutoff rigidities using sixth degree simulations of the geomagnetic field, J. Geophys. Res, 70, 4117-4130, 1965. 